\title{
Neuroprotective effects of a cardioplegic combination (adenosine, lidocaine, and magnesium) on an ischemic stroke model
}

Yichia Wang ( $\sim$ yichiawang@g.ntu.edu.tw)

National Taiwan University College of Medicine https://orcid.org/0000-0001-8021-5180

Yih-Sharng Chen

National Taiwan University College of Medicine

Sung-Tsang Hsieh

National Taiwan University College of Medicine https://orcid.org/0000-0002-3188-8482

\section{Research Article}

Keywords: Adenosine, lidocaine, and magnesium (ALM), cobalt chloride, ischemia, middle cerebral artery occlusion (MCAO), stroke

Posted Date: January 28th, 2022

DOI: https://doi.org/10.21203/rs.3.rs-1285972/v1

License: (c) (i) This work is licensed under a Creative Commons Attribution 4.0 International License. Read Full License 


\section{Abstract}

Adenosine, lidocaine, and magnesium (ALM) formula is a clinically available cardioplegic solution. We examined the effects of low dose ALM on ischemic stroke with cell and animal models. Cobalt chloride $\left(\mathrm{CoCl}_{2}\right)$-treated $\mathrm{SH}-\mathrm{SY} 5 \mathrm{Y}$ cells were used as our surrogate model to mimic oxygen-glucose deprivation condition. The cells were incubated with different dilutions of the ALM authentic solution (1.0 mM adenosine, $2.0 \mathrm{mM}$ lidocaine, $5 \mathrm{mM}$ MgSO4 in EBSS). At the concentration of 2.5\%, ALM significantly reduce $\mathrm{CoCl}_{2}$-induced cell loss. The protective effect remained if ALM was given 1 hour within the insult. We then used transient middle cerebral artery occlusion to investigate the therapeutic effects of ALM in vivo. Rats were randomly assigned to two groups: the experimental (ALM) group vs. control (saline) group, and infusion was given during the ischemia for one hour. The infarction area was significantly reduced in the ALM group $(5.0 \% \pm 2.0 \%$ vs. $23.5 \% \pm 5.5 \%, p=0.013)$. The neurological deficits was reduced in the ALM group (Modified Longa score: 0 [0-1] vs.2 [1-2], $p=0.047$ ). The neuroprotective effect was substantiated by the reduction of various neuron injury markers in the plasma. These results demonstrated the neuroprotective effects of ALM and may provide a new therapeutic strategy for ischemic stroke.

\section{Introduction}

Stroke is the second leading cause of death and disability in the world [1]. Despite the development of new medications, effective treatments for stroke at the acute stage is limited [2].A combination of adenosine, lidocaine, and magnesium (ALM) was first developed in 1998 to mimic hibernating state for cardiac protection in cardiac surgery [3]. Since adenosine and lidocaine both could cross the blood-brain barrier, ALM might offer neuroprotective effects to ischemic brain $[4,5] . I n$ cardiac arrest model with prolonged extracorporeal life support, ALM was associated with less neurological deficits, and lower tumor necrosis factor $a$ in the brain [3].

High dose ALM could arrest the heart contraction, and offer better myocardial protection than highpotassium depolarizing solutions. [6-8] At a low dose, ALM shows its protective effects in animal models of myocardial ischemia, arrhythmia, cardiac arrest, hemorrhagic shock, and septic shock [9]. The underlying mechanism could partly be explained by its ability to reduce inflammation, correct coagulopathy, and lower energy demands [10-12]. The protective effects of ALM on the cardiac system has been well elaborated [3]. However, it remains elusive whether the hibernating effects is beneficial to the brain. This issue has not yet been systematically examined.

Since ALM has never been studied in animal models of ischemic stroke before, it is difficult to evaluate ALM effect in clinical ischemic stroke. Critical clinical setting, infarction size, and reperfusion time are difficult to standardize for evaluation. Thus, we applied transient middle cerebral artery occlusion model to explore if ALM was helpful in ischemic braininjury with reperfusion. We hypothesized that the protective effects of ALM could decrease the damage of hypo-perfusion in ischemic stroke model; low 
dose ALM infusion would decrease infarction size. We first took advantage of a cell model and then animal model to explore this issue.

\section{Materials And Methods}

\section{Effects of cobalt chloride on the survival of SH-SY5Y cells}

Human neuroblastoma cell line,SH-SY5Y, was used in our study. The cells were grown to confluence in tissue culture wells with density $2 \times 10^{5}$. These cells were then maintained in culture with fetal bovine serum (FBS). After allowing the cells to differentiate in medium containing FBS for $24 \mathrm{~h}$, the medium was replaced with Earle's Balanced Salt Solution (EBSS) lacking glucose. After that, the cells were incubated in cobalt chloride $\left(\mathrm{CoCl}_{2}\right)$ with different concentration to reach oxygen deprivation via chemical methods. Cells were collected after 24 hours of incubation. Cell viability and hypoxic injury were analyzed with alamarBlue cell viability assay test and.HIF1-alpha expression respectively. Optimal oxygen and glucose deprivation condition was determined by reliable hypoxic damage.

\section{Cytotoxic profiles of ALM}

ALM (1.0mM adenosine, 2.0mM lidocaine, $5 \mathrm{mM}$ MgSO4) were diluted to $1.25 \%, 2.5 \%, 5 \%, 10 \%$, and $20 \%$ with EBSS solution to test its cytotoxicity in SH-SY5Y cell line. After adding ALM solution for 24 hours, we did alamarBlue cell viability assay to check cell survival.

\section{Effect of ALM treatment on cell viability}

We added ALM (1.0 mM adenosine, $2.0 \mathrm{mM}$ lidocaine, $5 \mathrm{mM} \mathrm{MgSO}_{4}$ )diluted to different concentrations by EBSS to $\mathrm{CoCl}_{2}$ incubated SH-SY5Y cells for 24 hours. We compared ALM treated groups to cobalt chloride incubated group, and determined the most effective therapeutic concentration for cell preservation. The protective effect of ALM was evaluated by alamarBlue cell viability test. In posttreatment group, we incubated SH-SY5Ycells in $50 \mu \mathrm{M}$ cobalt chloride, and then added ALM stock (1.0mM adenosine, $2.0 \mathrm{mM}$ lidocaine, $5 \mathrm{mM}$ MgSO4) diluted to $2.5 \%$ with EBSS solution in different time point after cobalt chloride treatment. Cell viability was analyzed with alamarBlue test.

\section{Animal preparation}

All animal experiments were carried out in conformity with the animal protocol approved by National Taiwan University College of Medicine and College of Public Health Institutional Animal Care and Use Committee (IACUC No. 20180302), and were conducted in compliance with the Animal Welfare Act (AWA), National Institute of Health Guide for the Care and Use of Laboratory Animals, and ARRIVE (Animal Research: Reporting in Vivo Experiments).A total of 12 Sprague Dawley rats (male, 7-8 weeks old, 235- 
250gm, BioLASCO Taiwan Co. Taipei, Taiwan) were studied. We investigating the effects of ALM treatment on infarction size following embolization.

In brief, rats were anesthetized with a $1.5 \%$ isoflurane-oxygen mixture by mask and after a short period of stability, anesthesia was reduced to $1.0 \%$ isoflurane for the remainder of the experimental period. Rectal body temperature was maintained between 37 and $38^{\circ} \mathrm{C}$ with a thermostatically regulated heating lamp. Body weights were recorded at baseline. Rats were placed in the prone position. Using aseptic surgical technique, the right femoral vein was cannulated for vascular access. The animals were housed in separate cages at ambient room temperature during recovery and thereafter until the end of the experiment.All animals were housed in a 14-10 hour light-dark cycle.

\section{Focal ischemia and surgical procedure}

Ischemia was produced by middle cerebral artery occlusion(MCAO) using the intraluminal suture technique [13]. Right common, external, and internal carotid arteries (CCA, ECA and ICA, respectively) were dissected from the surrounding connective tissue through a lateral neck incision. The filament was placed from right ECA and gently advanced via the ICA to approximately $20 \pm 0.5 \mathrm{~mm}$ from the carotid bifurcation.to middle cerebral artery. (filament total length $50 \mathrm{~mm}$; filament diameter $0.28 \mathrm{~mm}$; proximal silicon diameter $0.37+/-0.02 \mathrm{~mm}$; RWD Life science, Co.LTd).In the transient MCAO model, we left the filament in the ICA region for 1 hour, and then withdrew the filament to restore the blood flow.All experiments were performed by a single individual (YCW).

\section{Methodology}

The treatment modality, experiment group (ALM; $1.0 \mathrm{mM}$ adenosine, $2.0 \mathrm{mM}$ lidocaine, $5 \mathrm{mM} \mathrm{MgSO}_{4}$ in $0.9 \%$ normal saline) or control group ( $0.9 \%$ normal saline; $308 \mathrm{mOsm} / \mathrm{L})$, was assigned by random table, blinded in consecutively numbered sealed envelopes by another laboratory member who had the key. Treatment modality was blinded to the operator (YCW) until all the rats were sacrificed and results were collected.

After filament was placed in middle cerebral artery, we started to give treatment infusion by assigned number. In transient MCAO model, we kept the infusion rate by $2 \mathrm{ml} / \mathrm{kg} / \mathrm{hour}$ during ischemia for 1 hour. After the 1 hour infusion, the rats were allowed to recover from anesthesia in cage with free access of food and water.

\section{Neurological examination}

The rats were monitored for neurological deficits before the surgery and 24 hours after MCAO. Neurological deficits were determined using a modification of Longa scoring system as follows: 0 : no 
neurological deficit; 1: failure to extend right forepaw fully; 2: circling to the right; 3: falling to the right; and 4: did not walk spontaneously and had a depressed level of consciousness [14].

\section{Infarction size evaluationof brain}

The rats were sacrificed by isoflurane $24 \mathrm{~h}$ following MCAO and their brains were removed. The cerebrum was coronally sectioned (2-mm thick), and slices were stained with 1\% 2,3,5-triphenyltetrazolium chloride (TTC) solution (Sigma-Aldrich ${ }^{\circledR}$ ) at $37^{\circ} \mathrm{C}$ for 30 min [15]. We analyzed the infarction size by image J [16].

\section{Enzyme-linked immunosorbent assay (ELISA)}

After anesthesia, blood was collected before sacrifice by cardiac aspiration. The blood was transferred to tubes containing EDTA, and centrifuged at $1500 \mathrm{Xg}$ for 20 minutes twice. The supernatant, i.e. plasma, was measured for neuron-specific enolase (NSE), S100B, and matrix metallopeptidase 9 (MMP-9) level with ELISA (Elabscience) following the protocols of manufacturer.

\section{Statistical analysis}

All continuous variables were summarized as mean \pm standard deviation (SD), and categorical data was summarized by median and interquartile range. Data was tested for normality using the Shapiro-Wilk normality test, or by assessing Q-Q plots of residuals by GraphPad prism ( $v$ 9.3.1). In the cell model, cell viability test and HIF1a data were averaged by each treatment concentration, and analyzed using oneway analysis of variance (ANOVA) followed by Dunnett's post hoc comparison.

In the rodent model, the experimental unit is an individual animal, and the primary outcome was infarct volume. Sample sizes calculation for our study was based on alpha level 0.05 , power 0.8 , and expected $50 \%$ difference in experimental and control groups. Infarction size, plasma NSE, S100B, and MMP9 were compared with unpaired $t$ test. Neurological score was compared with Mann Whitney tests. A p-value $<0.05$ was considered significant throughout.

\section{Results}

\section{Effects of cobalt chloride $\left(\mathrm{CoCl}_{2}\right)$ on cell viability of differentiated SH-SY5Y cells}

To establish a cell model mimicking oxygen glucose deprivation, this study exposed differentiated $\mathrm{SH}$ SY5Y cells to $\mathrm{CoCl}_{2}$ of various concentrations for 24 hours according to the previously described protocols [17]. Cells maintained in the EBSS medium served as a control group. The number of differentiated SH-SY5Y cells were reduced with an alteration of morphology, i.e. from a fusiform pattern 
to a round shape (Fig. 1A). The change in cell viability was quantified and validated with the alamarBlue test. There was a substantial cell loss when $\mathrm{CoCl}_{2}$ concentration was larger than $50 \mu \mathrm{M}$.

\section{Expression of hypoxia-inducible factor 1a (HIF1 a) in cobalt chloride-treated SH-SY5Y cells}

To investigate whether cobalt chloride treatment can serve as a surrogate cell model of oxygen-glucose deprivation, we conducted western blotting on cell lysates of cobalt chloride-treatedSH-SY5Y cells. Hypoxia-inducible factor $1 \mathrm{a}(\mathrm{HIF} 1 \mathrm{a})$ expression was upregulated in $\mathrm{CoCl}_{2}$-stimulated $\mathrm{SH}-\mathrm{SY} 5 \mathrm{Y}$ cells with a dose-dependent manner (Fig. 2).

\section{Safety profiles of ALM}

To explore the safety profile of ALM, we incubated differentiated SH-SY5Y cells with various concentrations of ALM. The cell viability on adding ALM was similar to that of controls if the concentration of ALM was less than $10 \%$ (Fig. 3). This observation indicates that the feasibility of applying ALM with a concentration less than $10 \%$.

\section{Attenuation of $\mathrm{CoCl}_{2}$-induced neuronal cell death by ALM}

To study whether ALM was beneficial in $\mathrm{CoCl}_{2}$-induced neuronal cytotoxicity, we incubated differentiated SH-SY $5 Y$ cells with $50 \mu \mathrm{M} \mathrm{CoCl}_{2}$ and $\mathrm{ALM}$ of various concentrations (from $0-10 \%$ ). The cell viability quantified by alamarBluetest showed that $2.5 \% \mathrm{ALM}$ could significantly reduce $\mathrm{CoCl}_{2}$-induced loss (Fig. 4). We added 2.5\% ALM to differentiated SH-SY5Y cellsafter various durations $\mathrm{CoCl}_{2}$-treatment(from 0 to 6 hours). There were significant changes in morphology, i.e. neurites were lost and cell number was decreased in a time-dependent manner (Fig. 5B). The cell viability based on the alamarBlue test was significantly decreased if $2.5 \%$ ALM was given 2 hours after OGD began (Fig. $5 \mathrm{C}$ ).

\section{Reduction of the infarct area after transient MCAO by ALM}

To examine the therapeutic effects of ALM, we performed middle cerebral artery occlusion (MCAO) in rats. Rats were randomized into 2 groups: experimental (ALM) group vs. control (saline) group. The treatment was given during ischemia for 1 hour. Animals were sacrificed 24 hours later for examinations. The body weight and rectal temperatures were within normal limits in both ALM and saline groups. We measured neurological deficit 24 hours after recovery from the anesthesia with modified Longa score. The infarction area was significantly reduced in the ALM group compared to the control group (ALM: $5.0 \% \pm 2.0 \%$ vs. saline: $23.5 \% \pm 5.5 \%$, $p=0.013$, Fig. $6 \mathrm{~A}$ and $6 \mathrm{~B}$ ). Modified Longa score was significantly higher in the control group 24 hours after MCAO surgery (ALM: 0.0, [0-1] vs. saline: 2.0, [1-2], $p=0.047$, Fig. $6 \mathrm{C}$ ). Furthermore, we explored plasma markers of neuronal injury by measuring the concentration of neuron-specific enolase (NSE), S100B, and metalloproteinase 9 (MMP9) withenzymelinked immunosorbent assay (ELISA). The values were significantly lower in the ALM group compared to those in the saline group (NSE:ALM0.13 0.08 vs. saline: $0.36 \pm 0.08, p=0.02 ;$ S100B: ALM 75.28 \pm 5.1 vs. saline $123.8 \pm 19.81 ; p=0.03$;MMP9: ALM 7.06 \pm 0.57 vs. saline: $9.98 \pm 0.94, p=0.03$, Figure $6 D \sim F$ ) 


\section{Discussion}

Our study demonstrated two important findings. First, ALM exerted protective effects in cobalt chlorideinduced hypoxic cell model, and the protective effect remained effective if ALM was given 1 hour within the ischemic insult. Second, ALM infusion during ischemia decreased infarction size in the transient MCAO model.

Stroke is a leading cause of disability, and ischemic stroke caused by arterial occlusion is responsible for the majority of strokes [18]. The most effective treatment for ischemic stroke is reperfusion therapy byintravenous thrombolysis and endovascular thrombectomy[19]. However, revascularization have critical time period for treatment, and thus is not used universally in all ischemic stroke patients [20]. The other mainstream treatment for stroke is to preserve tissue viability including hypothermia [21, 22], enhancing collateral blood flow [23], controlling edema formation [24], and targeting specific molecules in ischemia-induced pathways $[25,26]$. But these managements do not show consistent clinical benefits [1].Development of safe and effective treatments is still a major challenge to experimental and clinical neuroscience. In this study, ALM showed its cytoprotection ability in SH-SY $5 Y$ cells exposed to $\mathrm{CoCl}_{2}$ as a surrogate model of oxygen and glucose deprivation. Furthermore, the infarction size was reduced in an ischemia and reperfusion rodent model of transient MCAO. ALM is already used clinically in cardiac surgery.[27] By adjusting its concentration, ALM may have potential roles in acute ischemic stroke treatment by neuroprotection.

Cobalt chloride has been used to induce hypoxic conditions in vivo and in vitro, because it activates HIF$1 \mathrm{a}$, causes mitochondrial damage, and increases reactive oxygen species generation after ischemia [28, 29]. In the current study, we used cobalt chloride to mimic hypoxic condition, and demonstrated the cytoprotective property of ALM. However, hypoxia in human brain causes damage to neuron model along with astrocyte, oligodentrocyte, and pericyte[30]. However the current cell model of SH-SY5Y did not examine the effects of ALM on neuroinflammation after ischemia given a lack of astrocyte and oligodendrocyte in the culture system. Further studies are required by exposing different cells to examine the cell preservation effects and mechanisms such as neuroinflammation.

Middle cerebral artery occlusion model is the most widely used model to mimic human focal ischemic stroke [31]. It produces focal occlusion of a large cerebral artery as seen in human stroke, and offers the opportunity to study the phenomenon after reperfusion [32]. Though the physiological variables and occlusion condition could be monitored and controlled with noninvasive means, such as Laser Doppler to reduce variability [33], blood flow to the posterior cerebral artery and branches of the internal carotid artery may be obstructed to different degrees during the procedure and lead to variable infarction area and size [34]. In addition, different histological staining methods could contribute to the inconsistency of infarct size measurement. 1\% 2,3,5-triphenyltetrazolium chloride (TTC) is a marker of tissue dehydrogenase and mitochondrial dysfunction and may overestimate infarct size[35]. To decrease the interference of drawbacks by MCAO model, we assigned our treatments with randomization and keep blinded until analysis. 


\section{Conclusion}

Low dose ALM could decrease brain infarct area in the stroke model of transient MCAO. Furthermore, the neuroprotective effect was substantiated by the reduction of various neuron injury markers in the plasma. These observations suggest the clinical potential of ALM in ischemia stroke which deserves further investigations.

\section{Declarations}

\section{Acknowledgements}

We would like to express our thanks to Prof. Thomas Toung who help us with rat ischemic model, Dr.TiYen Yeh for rat randomization and plasma preparation, and Dr. John-Chun Ho for cell model establishment.

\section{Funding}

The research leading to these results received funding from Ministry of Science and Technology in Taiwan under Grant Agreement No 109-2314-B-002-243-and 107-2314-B-002-043-MY3.

\section{Competing Interests}

The authors declare they have no financial interests.

\section{Author contribution statements}

All authors contributed to the study conception and design, material preparation, data collection and analysis were performed by Yi-Chia Wang, Yih-Sharng Chen and Sung-Tsang Hsieh. The first draft of the manuscript was written by Yi-Chia Wang, and all authors commented on previous versions of the manuscript. All authors read and approved the final manuscript.

Data availability: The datasets generated during and analysed during the current study are available from the corresponding author on reasonable request.

Ethics approval: All animal experiments were carried out in conformity with the animal protocol approved by National Taiwan University College of Medicine and College of Public Health Institutional Animal Care and Use Committee (IACUC No. 20180302)

Consent to participate: Not applicable

Consent for publication: Not applicable

\section{References}


1. Campbell BCV, De Silva DA, Macleod MR, Coutts SB, Schwamm LH, Davis SM, Donnan GA (2019) Ischaemic stroke. Nat Rev Dis Primers 5(1):70. doi:10.1038/s41572-019-0118-8

2. Matei N, Camara J, Zhang JH (2020) The Next Step in the Treatment of Stroke. Front Neurol 11:582605. doi:10.3389/fneur.2020.582605

3. Dobson GP, Letson HL (2016) Adenosine, lidocaine, and Mg2+ (ALM): From cardiac surgery to combat casualty care--Teaching old drugs new tricks. J Trauma Acute Care Surg 80(1):135-145. doi:10.1097/TA.0000000000000881

4. Santa-Maria AR, Walter FR, Valkai S, Bras AR, Meszaros M, Kincses A, Klepe A, Gaspar D, Castanho M, Zimanyi L, Der A, Deli MA (2019) Lidocaine turns the surface charge of biological membranes more positive and changes the permeability of blood-brain barrier culture models. Biochim Biophys Acta Biomembr 1861(9):1579-1591. doi:10.1016/j.bbamem.2019.07.008

5. Bynoe MS, Viret C, Yan A, Kim DG (2015) Adenosine receptor signaling: a key to opening the bloodbrain door. Fluids Barriers CNS 12:20. doi:10.1186/s12987-015-0017-7

6. Vinten-Johansen J (2013) Adenosine-lidocaine-magnesium non-depolarizing cardioplegia: moving forward from bench to bedside. Int J Cardiol 166(2):537-538. doi:10.1016/j.ijcard.2012.09.193

7. Owen CM, Asopa S, Smart NA, King N (2020) Microplegia in cardiac surgery: Systematic review and meta-analysis. J Card Surg 35(10):2737-2746. doi:10.1111/jocs.14895

8. Francica A, Vaccarin A, Dobson GP, Rossetti C, Gardellini J, Faggian G, Onorati F (2021) Short-term outcome of adenosine-lidocaine-magnesium polarizing cardioplegia in humans. Eur $\mathrm{J}$ Cardiothorac Surg. doi:10.1093/ejcts/ezab466

9. Granfeldt A, Letson HL, Dobson GP, Shi W, Vinten-Johansen J, Tonnesen E (2014) Adenosine, lidocaine and $\mathrm{Mg} 2+$ improves cardiac and pulmonary function, induces reversible hypotension and exerts anti-inflammatory effects in an endotoxemic porcine model. Crit Care 18(6):682. doi:10.1186/s13054-014-0682-y

10. Griffin MJ, Letson HL, Dobson GP (2014) Adenosine, lidocaine and Mg2+ (ALM) induces a reversible hypotensive state, reduces lung edema and prevents coagulopathy in the rat model of polymicrobial sepsis. J Trauma Acute Care Surg 77(3):471-478. doi:10.1097/TA.0000000000000361

11. Conner J, Lammers D, Holtestaul T, Jones I, Kuckelman J, Letson H, Dobson G, Eckert M, Bingham J (2021) Combatting ischemia reperfusion injury from resuscitative endovascular balloon occlusion of the aorta using adenosine, lidocaine and magnesium: A pilot study. J Trauma Acute Care Surg 91(6):995-1001. doi:10.1097/TA.0000000000003388

12. Letson HL, Dobson GP (2018) Adenosine, lidocaine, and Mg2+ (ALM) resuscitation fluid protects against experimental traumatic brain injury. J Trauma Acute Care Surg 84(6):908-916. doi:10.1097/TA.0000000000001874

13. Gubskiy IL, Namestnikova DD, Cherkashova EA, Chekhonin VP, Baklaushev VP, Gubsky LV, Yarygin KN (2018) MRI Guiding of the Middle Cerebral Artery Occlusion in Rats Aimed to Improve Stroke Modeling. Transl Stroke Res 9(4):417-425. doi:10.1007/s12975-017-0590-y 
14. Xu L, Ding L, Su Y, Shao R, Liu J, Huang Y (2019) Neuroprotective effects of curcumin against rats with focal cerebral ischemia-reperfusion injury. Int J Mol Med 43(4):1879-1887. doi:10.3892/ijmm.2019.4094

15. Shahjouei S, Cai PY, Ansari S, Sharififar S, Azari H, Ganji S, Zand R (2016) Middle Cerebral Artery Occlusion Model of Stroke in Rodents: A Step-by-Step Approach. J Vasc Interv Neurol 8(5):1-8

16. Schneider CA, Rasband WS, Eliceiri KW (2012) NIH Image to ImageJ: 25 years of image analysis. Nat Methods 9(7):671-675

17. Yoo SY, Yoo JY, Kim HB, Baik TK, Lee JH, Woo RS (2019) Neuregulin-1 Protects Neuronal Cells Against Damage due to CoCl2-Induced Hypoxia by Suppressing Hypoxia-Inducible Factor-1 alpha and P53 in SH-SY5Y Cells. Int Neurourol J 23(Suppl 2):S111-118. doi:10.5213/inj.1938190.095

18. Collaborators GBDS (2021) Global, regional, and national burden of stroke and its risk factors, 19902019: a systematic analysis for the Global Burden of Disease Study 2019. Lancet Neurol 20(10):795-820. doi:10.1016/S1474-4422(21)00252-0

19. Herpich F, Rincon F (2020) Management of Acute Ischemic Stroke. Crit Care Med 48(11):1654-1663. doi:10.1097/CCM.0000000000004597

20. Vitt JR, Trillanes M, Hemphill JC 3rd (2019) Management of Blood Pressure During and After Recanalization Therapy for Acute Ischemic Stroke. Front Neurol 10:138. doi:10.3389/fneur.2019.00138

21. Kuczynski AM, Marzoughi S, Al Sultan AS, Colbourne F, Menon BK, van Es A, Berez AL, Goyal M, Demchuk AM, Almekhlafi MA (2020) Therapeutic Hypothermia in Acute Ischemic Stroke-a Systematic Review and Meta-Analysis. Curr Neurol Neurosci Rep 20(5):13. doi:10.1007/s11910-02001029-3

22. Kuczynski AM, Ospel JM, Demchuk AM, Goyal M, Mitha AP, Almekhlafi MA (2020) Therapeutic Hypothermia in Patients with Malignant Ischemic Stroke and Hemicraniectomy-A Systematic Review and Meta-analysis. World Neurosurg 141:e677-e685. doi:10.1016/j.wneu.2020.05.277

23. Ma J, Ma Y, Shuaib A, Winship IR (2020) Improved collateral flow and reduced damage after remote ischemic perconditioning during distal middle cerebral artery occlusion in aged rats. Sci Rep 10(1):12392. doi:10.1038/s41598-020-69122-8

24. Yao Y, Zhang Y, Liao X, Yang R, Lei Y, Luo J (2020) Potential Therapies for Cerebral Edema After Ischemic Stroke: A Mini Review. Front Aging Neurosci 12:618819. doi:10.3389/fnagi.2020.618819

25. Howell JA, Bidwell GL 3rd (2020) Targeting the NF-kappaB pathway for therapy of ischemic stroke. Ther Deliv 11(2):113-123. doi:10.4155/tde-2019-0075

26. Lakhan SE, Kirchgessner A, Hofer M (2009) Inflammatory mechanisms in ischemic stroke: therapeutic approaches. J Transl Med 7:97. doi:10.1186/1479-5876-7-97

27. Francica A, Tonelli F, Rossetti C, Tropea I, Luciani GB, Faggian G, Dobson GP, Onorati F (2021) Cardioplegia between Evolution and Revolution: From Depolarized to Polarized Cardiac Arrest in Adult Cardiac Surgery. J Clin Med 10(19). doi:10.3390/jcm10194485 
28. Caltana L, Merelli A, Lazarowski A, Brusco A (2009) Neuronal and glial alterations due to focal cortical hypoxia induced by direct cobalt chloride (CoCl2) brain injection. Neurotox Res 15(4):348358. doi:10.1007/s12640-009-9038-9

29. Jones SM, Novak AE, Elliott JP (2013) The role of HIF in cobalt-induced ischemic tolerance. Neuroscience 252:420-430. doi:10.1016/j.neuroscience.2013.07.060

30. Tripathi VK, Subramaniyan SA, Hwang I (2019) Molecular and Cellular Response of Co-cultured Cells toward Cobalt Chloride (CoCl2)-Induced Hypoxia. ACS Omega 4(25):20882-20893. doi:10.1021/acsomega.9b01474

31. Lopez MS, Vemuganti R (2018) Modeling Transient Focal Ischemic Stroke in Rodents by Intraluminal Filament Method of Middle Cerebral Artery Occlusion. Methods Mol Biol 1717:101-113. doi:10.1007/978-1-4939-7526-6_9

32. Larpthaveesarp A, Gonzalez FF (2017) Transient Middle Cerebral Artery Occlusion Model of Neonatal Stroke in P10 Rats. J Vis Exp (122). doi:10.3791/54830

33. Liu F, McCullough LD (2014) The middle cerebral artery occlusion model of transient focal cerebral ischemia. Methods Mol Biol 1135:81-93. doi:10.1007/978-1-4939-0320-7_7

34. Komatsu T, Ohta H, Motegi H, Hata J, Terawaki K, Koizumi M, Muta K, Okano HJ, Iguchi Y (2021) A novel model of ischemia in rats with middle cerebral artery occlusion using a microcatheter and zirconia ball under fluoroscopy. Sci Rep 11(1):12806. doi:10.1038/s41598-021-92321-w

35. Liu F, McCullough LD (2011) Middle cerebral artery occlusion model in rodents: methods and potential pitfalls. J Biomed Biotechnol 2011:464701. doi:10.1155/2011/464701

\section{Figures}

\section{Figure 1}

Effects of cobalt chloride $\left(\mathrm{CoCl}_{2}\right)$ on survival of SH-SY5Y cells of $6.5,12.5,25,50,100$, and $200 \mu \mathrm{M}$ for 24 hours in comparison with controls of FBS and EBSS

(A) To mimic oxygen-glucose deprivation, differentiated SH-SY5Y cells were exposed to cobalt chloride for different concentrations with an increase in concentrations of cobalt chloride. There was a reduction of cell number and a change in morphology.

(B) The quantitatively viability assay of alamarBlue showed that cobalt chloride at the concentrations higher than $50 \mu \mathrm{M}$ significantly decreased the viability of differentiated SH-SY5Y cells

\section{Figure 2}


Upregulation of HIF1a protein expression in cobalt chloride-treated SH-SY5Y cells

Differentiated SH-SY5Y cells incubated with glucose deprivation solution and cobalt chloride for 8 hours showed upregulate HIF1a protein expression

\section{Figure 3}

Cytotoxic profiles of ALM

(A) To clarify the toxicity profile for ALM solution, we incubated differentiated SH-SY5Y cells with different concentrations of ALM for 24 hours.

(B) ALM concentration higher than $10 \%$ led to significant cell death according to the alamarBlue assay.

\section{Figure 4}

Effect of ALM-pretreatment on cell viability

(A) We incubated differentiated SH-SY5Y cells with $50 \mu \mathrm{M}$ cobalt chloride, and added various concentrations of ALM for 24 hours.

(B) Pretreatment with ALM of the optimal concentration preserved the differentiated SH-SY5Y cells.

(C) The cell viability test of alamrBlue demonstrated that ALM 2.5\% solution was the optimal concentration to attenuate cell loss on incubation with EBSS and cobalt chloride.

\section{Figure 5}

Effect of ALM treatment on the rescue of cell viability after various cobalt chloride incubation duration

(A) The diagram depicted the study design to assess the effect of ALM after cobalt chloride $\left(\mathrm{CoCl}_{2} 50 \mu \mathrm{M}\right)$ treatment for various durations (from 1 hour to 6 hours).

(B) The number of differentiation SH-SY5Y cells decreased if ALM treatment was given after longer cobalt chloride incubation period: (B1) differentiated SH-SY5Y cells with $50 \mu \mathrm{M} \mathrm{CoCl2;} \mathrm{(B2)} \mathrm{ALM} \mathrm{2.5 \%}$ given together with $\mathrm{CoCl} 2$; (B3) ALM 2.5\% given after $\mathrm{CoCl} 2$ incubation for 1 hours; (B4) ALM 2.5\% given after $\mathrm{CoCl} 2$ incubation for 2 hours; (B5) ALM 2.5\% given after $\mathrm{CoCl} 2$ incubation for 4 hours; (B6)ALM $2.5 \%$ given after $\mathrm{CoCl} 2$ incubation for 6 hours 
(C) The beneficial effect of ALM disappeared if the treatment of ALM was given later than 2 hours after $\mathrm{CoCl} 2$ incubation according to the viability alamarBlue test.

\section{Figure 6}

Effect of ALM on a rodent stroke model of transient middle cerebral artery occlusion (MCAO)

(A) The infarct areas were measured 24 hours after MCAO by 1\% 2,3,5-triphenyltetrazolium chloride (TTC) solution.

(B) Infarction area was significantly larger in saline group than that in the ALM group

(C) Neurological deficits were measured with modified Longa score before MCAO and 24 hours after MCAO before sacrifice. The modified Longa score was the same in ALM and saline groups before surgery, and saline group scored higher than ALM group 24 hours after MCAO procedure.

(D) Plasma level of neuron specific enolase (NSE) quantified with ELISA was higher in the saline group than in the ALM group

(E) Plasma level of S100B quantified with ELISA was higher in the saline group than in the ALM group

(F) Plasma level of metalloproteinase 9 (MMP9) quantified with ELISA was higher in the saline group than in the ALM group 